\title{
Direct-to-Consumer Advertising: Should There Be a Free Market in Healthcare Information?
}

\author{
ANDREAS HASMAN and SØREN HOLM
}

On June 3, 2003, the European Council of health ministers rejected a proposal from the European Commission to allow drug manufacturers to advertise directly to particular groups of patients; the proposal had already been rejected by the European Parliament subsequent to a heated public debate in which consumer and patient groups almost unanimously argued that it was not the role of drug companies to provide information to patients. The pilot scheme suggested by the Commission would only have applied to patients with three chronic diseases, AIDS, diabetes, and asthma, and would, it was argued, not undermine an overall ban. Drug companies would have been required to abide by a special code of conduct and clear any information given to patients - on web sites or in specialized publications - with national authorities.

In parallel with these developments, the European Commissioner for Enterprise and Information Society Erkki Liikanen and Public Health Commissioner David Byrne launched the so-called group of ten or "G10." This was an initiative that brought together representatives of patients, national governments, and the drug and health insurance industries to discuss ways of increasing the competitiveness of the European pharmaceutical industry and capitalizing on the industry's potential for improving public health in the European Union. One issue that was discussed was the possibility of lifting the current ban on direct-to-consumer advertising of prescription-only medicines (DTCA) in order to extend the information on healthcare available to patients. In light of political developments, it was not surprising that in June 2004 the G10 recommended that a clear distinction be made between patient information and commercial marketing of pharmaceutical products, and that the ban on DTCA should remain in force.

One of the G10 members who most explicitly supported this recommendation was Angela Coulter. ${ }^{1}$ Amid widespread enthusiasm for liberalization of drug advertising among other health commentators, she argued convincingly that it is inappropriate to delegate responsibility for informing patients to commercial companies, that marketing typically is biased and untruthful, and that a free market for information about medicines would fail to empower patients to make independent decisions about their own care and treatment.

Coulter's argument is largely representative of those opposed to lifting the ban on DTCA and this article takes her position as a starting point. We point out that the evidence on whether DTCA should be allowed in a regulated form or banned is divided. From this preliminary conclusion we proceed to introduce further issues into the debate, which point to the advantages of a much 
softer approach, entailing selective regulation, rather than a blanket ban on advertising.

\section{For and Against a Free Market in Information}

A number of senior policymakers and health commentators have expressed support for the idea of giving the pharmaceutical industry liberty to inform patients directly about treatment options on offer. ${ }^{2}$ A typical line of argument in favor of lifting the ban on DTCA is that in order to make informed choices for themselves, patients require more information about the options for treatment available, and that the most efficient way to achieve this is to let the producers raise public awareness about their products. ${ }^{3}$ There is a perception that healthcare systems in general, and doctors in particular, have so far been insufficiently proactive in providing patients with information, and that many health problems, for which treatment is available, go unrecognized in their early phases. Other resources and new approaches will have to be brought into play to promote disease awareness in the general public, especially in groups that are underutilizing health services at present. It is also argued that because the industry under the current ban has had to target their information efforts at healthcare professionals (i.e., doctors), they have de facto contributed to the widening of a perceived "knowledge gap" between suppliers and consumers of healthcare services. A doctor's monopoly on information and knowledge about health and healthcare has contributed to an increase in patients' dependency on the medical profession and has reinforced paternalistic practices. Removing the ban on DTCA would, arguably, help to rectify this imbalance.

Evidence from New Zealand and the United States, where DTCA is already sanctioned, lends some support to these conclusions. Sumpradit et al. ${ }^{4}$ explored how chronic patients' interactions with doctors were affected by DTA and found that a positive attitude toward advertising among patients was associated with their willingness to talk with doctors about the advertised drugs. Crucially, those patients who eventually asked for a prescription tended to agree that DTCA helped them make their own decisions about medical treatment. Murray et al. ${ }^{5}$ found that DTCA strengthens patient-doctor relationships because it encourages patients to disclose health concerns to their doctors and enhances patients' sense of control and self-efficacy during the consultation. Doctors also find DTCA advantageous to the relationship, provided that they find the information conveyed in advertisements relevant and adequate, and that patients seek their opinion rather than demand a specific treatment. The positive impact of DTCA on the patient-doctor relationship seems most significant in groups of lower socioeconomic status and doctors, which could make a concern for equity an argument in favor of lifting the ban. ${ }^{6}$

Research also shows DTCA to be an effective way of targeting clinical problems, which are generally underdiagnosed in the population. Thus, whereas DTCA is generally market expanding in underdiagnosed therapeutic classes, it seems to have little or no influence on the brand of medicine chosen (no business-stealing effect); that supports the claim that marketing reaches patients who would otherwise lack information. ${ }^{7}$ Further study has shown, moreover, that DTCA effectively increases the number of requests that patients from lower socioeconomic groups, who are traditionally considered hard to reach with public health campaigns, make for preventive treatment and sched- 
uled check-ups. ${ }^{8}$ DTCA may therefore be an efficient way of changing health behavior and improving health utilization. At the same time there is a strong incentive for the pharmaceutical industry to advertise. In the United States, every \$20 invested in DTCA generates one new prescription with an average wholesale price that far exceeds that. There seems to be few losers if DTCA is deregulated. The fact that advertising raises public awareness about treatment in areas where better information is scarce, and at the same time increases the industry's profitability, has led some economists to go as far as to call DTCA "a public good." 9

Coulter rejects these arguments on the grounds that the health information that can be made available to patients through commercial marketing is inadequate, biased, untruthful, and restricted mainly to blockbuster drugs. She argues that regardless of the fact that there is a need for the public to become better informed about medical treatment and health issues in general, it should not be the responsibility of the drug industry to deliver this information. Commercial companies will seek maximum profit and will tend to exaggerate the significance of those health problems for which they offer a medical cure. Their marketing efforts will encourage scared patients to demand medicine that is "not clinically needed" and deceive them into believing that they suffer from serious conditions that would otherwise not have caused them any concern. ${ }^{10}$ This will introduce a new relationship of dependency where patients are reliant on the producers of medicines, and it will distort priorities because patients will pressure doctors to prescribe drugs that have been promoted by the industry.

She also finds that the information that is offered by the industry is of a substandard quality and gives patients an unrealistic impression of what they can expect from their treatment. Rather than giving patients access to comprehensive, unbiased information on the pros and cons of a given treatment, advertising is inherently partial, single-sided, and unambiguously positive toward one product or brand. It is, after all, not in a company's interest to inform consumers that a competitor has a superior product-even if this indeed is the case. Due to this lack of information, patients will supposedly lose out.

Coulter acknowledges that paternalism remains a significant problem in healthcare delivery, and that the lack of information available to patients is seriously counteracting any improvement in medical practice on this point. She argues, however, that allowing DTCA would not have the effect opponents of the ban hope for, because it would not address the substance of the problem. Information from drug companies would, allegedly, be seen as "poor quality information" by both patients and doctors and would do nothing to help identify the patient's true preferences and options within the clinical consultation. Doctors would know that advertisers are attempting to manipulate patients into requesting their products and that could work against patients.

Instead of allowing DTCA of prescription medicines, Coulter suggests a partnership involving the government, patients, government agencies, and the industry. Such a joint effort would, she argues, be the only way to ensure that consumers get balanced information of high quality about the effects and adverse effects of medicines. Drawing together a wider range of resources, the partners would be able to inform the public as well as patients about health and healthcare, based on the best available evidence and current practice.

Coulter's claims are also supported by evidence. In New Zealand $40 \%$ of spending on DTCA is used to promote 10 medicines, which are all new, 
expensive patented drugs for long-term treatment of conditions such as allergy, ulcer, anxiety, obesity, impotence, and high blood cholesterol. ${ }^{11}$ This finding is explained in other research that shows that pharmaceutical companies are much less likely to actively promote a drug to consumers if their competitors offer the same or a very similar product, and that "first move advertisers" are likely to spend more money on DTCA than those competitors who start to advertise later. ${ }^{12}$ The effect is that DTCA will naturally be concentrated on a few branded blockbuster drugs. More worryingly perhaps, studies in the United States have shown that drug advertisements tend to play on consumers' anxieties and fears of serious illness, promote the impression that medicine is the only solution to health problems, ${ }^{13}$ and give confusing or incorrect information about risk factors, disease prevalence ${ }^{14}$ and side-effects. ${ }^{15}$ Prescribing doctors experience the public's anxieties as a direct pressure, and evidence confirms that physicians feel compelled to prescribe medicines that have been actively promoted in the media. In a telephone poll carried out among prescribing doctors in Ohio, $91 \%$ of respondents reported that they did feel pressured by requests from patients and that in $36 \%$ of such cases doctors gave in to patients and prescribed a drug that would not have been their first choice of treatment. ${ }^{16}$ Other studies have shown DTCA to have an adverse effect on doctors' perceptions of time efficiency and the number of appropriate tests and treatments carried out, ${ }^{17}$ and that doctors are more likely to feel their authority and clinical judgment challenged if patients refer to information from advertisements. That perception is potentially harmful (even if it is mistaken) to the relationship between patients and doctors because it undermines the reciprocated trust that is so essential to a successful interaction between them. ${ }^{18}$ Health insurers in the United States have, moreover, long been concerned about the cost implications of increasing demand and a shift in prescription patterns toward expensive preventive drug treatments aimed at broad segments of the population. DTCA is believed to be at least part of the reason for historic hikes in U.S. pharmaceutical spending in the last decade. ${ }^{19}$

\section{Free Markets and Prescription Medicines}

Based on the literature review above, the evidence on DTCA appears inconclusive, and seems to provide support to both those proposing and opposing deregulation of the advertising market for new medicines. The policy decision cannot be made on evidence alone. One key premise that seems to underpin the call for the abolition of the ban is the understanding that free competition in an unregulated market is, prima facie, the most efficient way for consumers to get information about drug options and to eventually satisfy their preferences for medical treatment. Those opposed to lifting the ban on DTCA may want to dismiss the viability of this premise by rejecting the overall advantages of advertising and free competition - but then the argument against DTCA would inevitably have consequences far beyond the market in prescription medicines. It would infer a rejection of a market economy as such, and that is probably not what Coulter and others in favor of a ban on DTCA want to advocate. Instead they may well accept the premise in principle but argue that, although it is relevant in other sectors of the economy, the principle does not apply to the market for prescription drugs. Accepting the premise that there is an advantage to having free unregulated markets does, of course, not necessarily imply a 
rejection of any kind of market regulation. The workings of the particular market may indeed be such that a ban is justified. The onus is clearly on those who want a ban, however, to provide convincing justification for such a policy.

The market for medical treatment is different from most other markets in the economy, because it is characterized by deep asymmetries in information. In the market for medical treatment it is often the case that the supplier of healthcare has much better information about what the buyer needs than the buyer has. This means that it is easy for suppliers to induce demand for their services, and that some of the usual assumptions about informational symmetry in free markets cannot be upheld in the medical market. ${ }^{20}$ We may argue that regulation of the market intended to minimize supplier-induced demand can therefore be justified. It is, however, important to note that the supplier best placed to induce demand in the medical market is the healthcare professional who is in direct contact with the patient, and that informational asymmetries between suppliers and consumer of drugs are usually considerably smaller.

\section{A Ban on Free Information}

Can the asymmetries in the market for healthcare information provide sufficient grounds for regulation in the form of an outright ban on DTCA of prescription drugs? Coulter's reasons for supporting such a ban can be summarized as follows: DTCA is inherently poor quality information; DTCA undermines the patientdoctor relationship and increases patients' dependency on drug producers; DTCA distorts clinical priorities and increases the demand for and cost of healthcare. But are these reasons strong enough to justify a complete ban?

It is not surprising that drug advertising is single-sided and biased in favor of a particular product (after all, advertisement usually is). There are at least three groups of patients, however, who may prefer the simple and easily accessible information offered in advertisements to the more comprehensive, balanced, and technical information entailed in Coulter's partnership approach. First, there is a significant group of patients of low socioeconomic status, whom it is notoriously difficult to reach with traditional health information. Because DTCA has proved to be a more effective way of informing this group, it seems fair to argue that although the information conveyed in advertising is not completely exhaustive, it is still better than no information. Banning advertising may in some cases leave these patients worse off. For example, if I am not aware that modern and reasonably effective drugs against urge incontinence are available, I may unnecessarily continue to live with my incontinence and my incontinence pads. Second, there is a group of patients suffering from minor or temporary health problems who, although capable of accessing more complex information, still prefer the easily available information from advertising. They may find that their time is better spent on other things, and that DTCA gives them sufficient information to ask for a relevant drug when visiting their doctor. An example of a patient from this group would be the busy professional who can recognize the first symptoms of an ulcer from a drug advertisement and can therefore request an appropriate drug from her doctor. Even in the cases where the advertised drug is not entirely appropriate, advertisement will at least have initiated an exchange of information between patient and doctor. Third, there is a group of patients who have extensive experience with managing chronic, long-term conditions or recurring illness. 
They may find that they already have all the information they require to make informed decisions and that they should be allowed a free choice of a drug with a branded image if they so wish. In the choice of asthma inhaler the chronic sufferer may, for instance, emphasize ease of use or smart design or compatibility with this season's colors in choosing between inhalers with very similar efficacy. It is difficult to see why people should be denied this information, which is probably unlikely to figure prominently in governmentcontrolled health information. Increased demand from these groups, to which most patients probably belong, will not be due to information asymmetry between users and producers of drugs. In fact, denying them information from advertisements will potentially harm some patients, because it exposes them to exploitation by health professionals who can take advantage of the information gap that persists between them and patients. This is not to say, of course, that there are not patient groups who may be unduly influenced by commercial advertising and should be protected from the adverse effects of DTCA. What it does mean, however, is that there are clear adverse effects of an outright ban.

The argument that DTCA evidently increases demand for only a small number of heavily promoted categories of drugs carries some merit, but it also seems insufficient justification for prohibiting advertising. Although the argument points to the need for supplementary information being made available alongside information from advertisements, it does nothing to disqualify the quality of the information provided by DTCA. Increased demand may well be due to patients' improved ability to choose medicines in accordance with their preferences, based on sufficient and appropriate information. If that is the case, then "disease mongering" is maybe not such a bad thing after all. The fact that members of the medical profession see a given problem as "an ordinary ailment" for which treatment is "not clinically needed" 21 is essentially irrelevant if patients find the problem significant enough to seek treatment. It is therefore not a problem that doctors are unhappy about patients demanding specific treatments and feel the patient-doctor relationship undermined by DTCA. Doctors should get into the habit of arguing their case if they think that what the patient requests is clinically inappropriate. This would, over time, strengthen rather than weaken the relationship. There will, of course, be some drug categories that are not promoted in advertisements. Information on cheap and established generic medicines, such as, for example, low dose aspirin, is unlikely to be available through DTCA, and alternative ways to raise public awareness about these treatments must be found. But this highlights a need for healthcare information in addition to the information available through DTCAnot the abolition of DTCA.

That advertising drives up the cost of healthcare provision is a distinct issue that relates to problems of "moral hazard" in insurance systems. Copayment schemes can be and are used to address this. It obviously does not follow directly from allowing DTCA for certain drugs that a public healthcare system should still fund prescriptions in the same way as in areas without DTCA. This is a separate issue.

\section{Why a Partial Ban?}

The asymmetries in the market for prescription drugs may justify some degree of intervention by government agencies. A balanced approach is nevertheless 
required. On the one hand, it cannot be left entirely to the drug industry to inform patients about medicines because information will then only be available on some medicines and because some patients do not get the information they need in order to make independent decisions from advertising. Alternative information resources should, therefore, we might argue, be made available to them. On the other hand, however, there are some groups of patients who are effectively harmed as a direct result of a ban on DTCA, either because they need information that is only available through advertising or because they simply prefer the more easily accessible information offered in advertisements. For this reason, a more selective approach to regulation of DTCA is preferable to a blanket ban.

It is also important to note that the alternative to allowing DTCA is not the provision of unbiased and truthful information. Implying or stating a dichotomous choice between DTCA or unbiased and truthful information is simply a false dichotomy. As noted above, there is a general lack of health information and a general lack of penetration of the available information to disadvantaged groups. We may hope that governments will take their responsibility for providing health information more seriously in the future, but it is difficult to see a direct link between this and the DTCA issue. It is also obvious that whereas at least one pharmaceutical firm has an interest in promoting the most effective treatment, a public healthcare system only has an interest in promoting the most cost-effective treatment (which may or may not be the most effective). We can therefore not assume that continuing a blanket ban on DTCA will automatically lead to the adequate provision of unbiased and truthful information.

Our conclusion is, therefore, that, on balance, it is difficult to see why DTCA should be banned outright. It seems fair to argue that a regulated market for healthcare information provided by suppliers to consumers should be allowed in areas such as (1) chronic disease, in which patients usually develop expertise in understanding and managing their own condition (asthma, diabetes, etc.); (2) minor or short-lived health problems that do not require patients to have extensive information to make decisions about treatment efficiently (early symptoms of ulcer, etc.); (3) undertreated patient groups in which initial self-diagnosis is sufficiently reliable and not too worrying (incontinence, hair loss, etc.); and (4) prescription areas with a large life-style component (impotence, mood enhancement, etc.). We do not claim that this list is exhaustive, and each item on the list also needs further elaboration. We do, however, claim that for the disease categories and treatment modalities on our list the burden of proof falls strongly on those who want a ban on DTCA to remain in place. A system that allows for DTCA in some areas of the market for prescription medicines yields clear rewards, and the European Commission should strive to pass legislation that gives patients the opportunity to enjoy the benefits of free healthcare information, while at the same time protects those patients who are particularly vulnerable against its disadvantages.

\section{Notes}

1. Coulter, A. Why the ban on direct-to-consumer advertising of prescription medicine should remain in place. In: Asato J, ed. Direct to Patient Communication: Patient Empowerment or NHS Burden? London: Social Market Foundation; 2004:37-43. 


\section{Direct-to-Consumer Advertising}

2. Evans T. The state, the market and freedom of health information. In: Asato J, ed. Direct to Patient Communication: Patient Empowerment or NHS Burden? London: Social Market Foundation; 2004:32-6; Colin-Thome D. Empowering patients through access to information. In Asato J, ed. Direct to Patient Communication: Patient Empowerment or NHS Burden? London: Social Market Foundation; 2004:10-5.

3. Holmer AF. Direct-to-consumer advertising-strengthening our health care system. New England Journal of Medicine 2002;346:526-8.

4. Sumpradit N, Fors SW, McCormick L. Consumers' attitudes and behavior toward prescription drug advertising. American Journal of Health Behavior 2002;26(1):68-75.

5. Murray E, Lo B, Pollack L, Donelan K, Lee K. Direct-to-consumer advertising: Public perceptions of its effects on health behaviors, health care, and the doctor-patient relationship. Journal of the American Board of Family Practice 2004;17:6-18.

6. Murray E, Lo B, Pollack L, Donelan K, Lee K. Direct-to-consumer advertising: Physicians' views of its effects on quality of care and the doctor-patient relationship. Journal of the American Board of Family Practice 2003;16:513-24.

7. Iizuka T. What explains the use of direct-to-consumer advertising of prescription drugs? The Journal of Industrial Economics 2004;52(3):349-79.

8. See note 5, Murray et al. 2004, and Understanding the Effects of Direct-to-Consumer Prescription Drug Advertising. Menlo Park, Calif.: Henry J. Kaiser Foundation; 2001.

9. Iizuka T, Jin GZ. The effects of direct-to-consumer advertising in the prescription drugs markets. Working paper, Nashville, Tenn.: Vanderbilt University; 2003.

10. Moynihan R, Heath I, Henry D. Selling sickness: The pharmaceutical industry and disease mongering. British Medical Journal 2002;324:886-91.

11. Mintzes B, Barer ML, Kravitz RL, Kazanjian A, Bassett K, Lexchin J, et al. Influence of direct to consumer pharmaceutical advertising and patients' requests on prescribing decisions: Two site cross sectional survey. British Medical Journal 2002;324:278-9.

12. See note 7 , Iizuka 2004.

13. Mintzes B. Blurring the boundaries: New trends in drug promotion. Amsterdam: Health Action International; 1998, available at: http://www.haiweb.org/pubs/blurring/blurring.intro.html.

14. Consumer Association. Promotion of Prescription Drugs: Public Health or Private Profit? London: Consumers' Association; 2001.

15. See note 8, Understanding the Effects of Direct-to-Consumer Prescription Drug Advertising 2001.

16. Spurgeon D. Doctors feel pressurised by direct to consumer advertising. British Medical Journal 1999;319:1321, and see note 11, Mintzes et al. 2002.

17. See note 6, Murray et al. 2003.

18. See note 6, Murray et al. 2003.

19. Danzell MD. Direct-to-consumer advertising: Can everyones's interests be balanced? Managed Care 1999 Dec, available at: www.managedcaremag.com/archives/9912/9912.dtc.html (accessed July 2004).

20. Arrow KJ. Welfare economics of medical care. The American Economic Review 1963;53(5):941-73.

21. See note 10, Moynihan et al. 2002. 